\section{Tellurite Reduction in Yersinia}

In an attempt to find further biological differences between Yersinia malassezii (Pasteuvella pseudotuberculosis) and $Y$. enterocolitica, the growth of strains of the latter group on tellurite medium was observed. It has already been reported ${ }^{1}$ that $P$. pseudotuberculosis does grow on tellurite media. Now some other strains of this group were used for this comparative study.

Ten strains of $Y$. entevocolitica and 14 strains of $P$. pseudotuberculosis (type I-V) were obtained through the courtesy of Dr. H. H. Mollaret, Paris.

The Clauberg tellurite medium was used. Before testing, the strains of both groups were subcultured twice on $5 \%$ bovine blood agar. In the tests the inoculum was large and was made as equal as possible in both groups. The incubation was at $37^{\circ} \mathrm{C}$ for $48 \mathrm{~h}$.

All strains of $P$. pseudotuberculosis grew to visible colonies on the tellurite medium, whereas the strains of $Y$. enterocolitica did not. Only occasional small colonies appeared in some strains of $Y$. enterocolitica during the second half of the $48 \mathrm{~h}$ incubation in the repeated experiments with still larger inoculums.

The growth of all strains of $P$. pseudotuberculosis on tellurite medium was abundant also in repeated experiments with smaller inoculums. The only exception was the strain 52, type V (strain 25 of Thal) the growth of which was feeble and delayed. It will be described more extensively elsewhere.

So far, the observations seem to indicate that $Y$. enterocolitica is devoid of tellurite reductase whereas this enzyme is present in $P$. pseudotuberculosis.

Comparative studies should be extended to other organisms of the group, especially to $Y$. pestis, because the results might prove useful for the differential diagnosis of these and related bacteria ${ }^{2}$.

Zusammenfassung. In Gegensatz zu Yersinia malassezii scheint $Y$. enterocolitica keine Tellurit-Reduktase zu besitzen.

B. BRzIN

Institute of Microbiology, Ljubljana (Yugoslavia), 10 October 1967.

1 B. Brzin, Zentbl. Bakt. Parasit Kde 189, 543 (1963).

2 My thanks are due to Dr. H. H. Mollaret for giving the strains of Pasteurella pseudotuberculosis and Yersinia enterocolitica at my disposal.

\title{
COGITATIONES
}

\section{Triterpènes $X I^{1}$. Fréquence et site d'oxygénation des triterpènes naturels}

Les remarques qui suivent portent sur la comparaison de 94 représentants naturels du groupe de la $\beta$-amyrine et de 73 triterpènes tétracycliques ${ }^{2}$ connus au début de 1967. Les tétracycliques seront, pour notre présent propos, considérés globalement, sans distinction de sous-groupes. Les triterpènes pentacycliques des groupes de l' $\alpha$-amyrine, du lupéol et a fortiori des groupes mineurs ne seront pas envisagés ici, le nombre relativement restreint de leurs représentants connus ne permettant pas encore de généralisation, quoiqu'ils semblent s'intégrer dans l'ensemble des données ci-dessous rapportées.

Il nous a paru intéressant de calculer le pourcentage des triterpènes naturels oxygénés en chacune des positions du squelette et de comparer les résultats obtenus dans les 2 séries en faisant correspondre les atomes de carbone biogénétiquement équivalents.

Tous les triterpènes $\beta$-amyréniques et $92 \%$ des tétracycliques $^{3}$ sont oxygénés en $\mathrm{C}-3$. Cette prédominance bien connue est en accord avec la structure du précurseur commun actuellement admis, le 2,3-oxydosqualène ${ }^{4}$.

Les 2 séries diffèrent principalement en ce que $21 \%$ des $\beta$-amyréniques sont oxygénés en $\mathrm{C}-23$, fait dont on ne retrouve aucunement l'équivalent chez les triterpènes tetracycliques où, dans le groupe lanosténique, l'oxygénation des méthyles portés par $\mathrm{C}-4$ semblerait mener rapidement, par attaques ultérieures, à la série stéroïdique ${ }^{5}$. On constate, d'autre part, que le carbone C-20 est oxydé chez $22 \%$ des triterpènes tétracycliques, alors que son homologue $\mathrm{C}-17$, quaternaire en série $\beta$-amyrénique, ne pourrait l'être.
Venant après le carbone $\mathrm{C}-3$, la position la plus fréquemment oxygénée en série $\beta$-amyrénique est $\mathrm{C}-28$ $(71 \%)$, dont les voisins $\mathrm{C}-16$ et $\mathrm{C}-22$ sont oxydés à raison de $34 \%$ et $24 \%$, respectivement. Il en va de même pour leurs équivalents biogénétiques C-21, C-16 et C-22 en série tétracyclique, oxygénés à raison de $23 \%, 14 \%$ et $7 \%$. Il nous faut constater, en outre, que les fréquences relatives d'oxygénation de ces positions sont très semblables dans les 2 groupes (environ 10/5/3,5 en série $\beta$-amyrénique et $10 / 6 / 3$ en série tétracyclique) bien que leur disposition - et partant leur accessibilité - soit nettement différente.

Une rationalisation possible de ces observations serait d'admettre que l'oxygénation privilégiée du C-28 (et, peut-être, de $\mathrm{C}-16$ et $\mathrm{C}$-22) des triterpènes $\beta$-amyréniques

I Partie XV. B. Tursch, D. Daloze, C. Bartholomé et G. ChiurDoglu, Bull. Soc. chim. Belg., à paraître.

2 A l'exception du groupe de la limonine et de ses analogues hautement oxygénés.

3 La numérotation adoptée ici est celle de P. BoIteav, B. Pasici et R. Ratsimamanga, Les Triterpénoides en Physiologie végétale et animale (Gauthiers-Villars Paris, 1964).

4 E. J. Corey, W. E. Russey et P. R. Ortiz de Montellano, J. Ain. chem. Soc. 88, 4750 (1967) et F. E. Van Tammelen, E. E. Willett, R. B. Clayton et K. E. Iorn, J. Am. chem. Soc. $88,4752(1967)$.

5 Pour une revue récente voir R. B. Cuayton, Q. Rev. chem. Soc. 19,168 et 201 (1965). 